\title{
A methodology to enable automatic 3D routing of aircraft Electrical Wiring Interconnection System
}

\author{
Z. Zhu' ${ }^{1}$ • G. La Rocca ${ }^{1}$ M. J. L. van Tooren ${ }^{2}$
}

Received: 29 February 2016/Revised: 8 December 2016/Accepted: 20 February 2017/Published online: 10 March 2017

(C) The Author(s) 2017. This article is published with open access at Springerlink.com

\begin{abstract}
Harness 3D routing is one of the most challenging steps in the design of aircraft Electrical Wiring Interconnection System (EWIS). This is due not only to the intrinsic complexity of the EWIS, but also to the increasing number of applying design constraints and its dependency on any change in the design of the airframe and installed systems. The current routing process employed by EWISdesign is largely based on the manual work of expert engineers, partially supported by conventional CAD systems. As a result, the routing process is quite inefficient, error prone and unable to deliver optimal solutions. Although many harness components are selected from catalogues and the design process is largely repetitive and rule based, it has been found that none or very limited automation solutions, which can significantly decrease the workload of engineers and increase their efficiency, are currently available. In this paper, an innovative approach is proposed to solve the 3D routing automation as an optimization problem. Knowledge Based Engineering (KBE) and optimization methods are proposed to achieve minimum cost routing solutions that satisfy all relevant design rules and constraints. The proposed solution is scalable in terms of constraints, can be deployed on any type of routing environment, and, thanks to the achieved level of automation, able to reduce the process lead time drastically. The basic idea is to
\end{abstract}

This paper is based on a presentation at the CEAS Air \& Space Conference 2015, September 7-11, Delft, The Netherlands.

\section{Z. Zhu}

z.zhu@tudelft.nl

1 Faculty of Aerospace Engineering, Delft University of Technology, Delft, Netherlands

2 College of Engineering and Computing; Ronald E. McNAIR Center for Aerospace Innovation and Research, University of South Carolina, Columbia, SC, USA achieve optimal EWIS routing solutions by optimizing the position of the harnesses clamping points, which are used as way-points to route the harnesses inside the aircraft digital mock-up. The challenge to solve this optimization problem is that the number and initial value of design variables, namely the number and position of clamping points, are not known a priori. To handle this challenge, a two-step, hybrid optimization strategy has been devised. The first step, called Initialization, uses a road map based path finding method to generate a preliminary harness definition, including the required number and preliminary position of its clamping points. The second step, called Refinement, uses a conventional optimization method to move the position of the clamps and refine the preliminary harness definition aiming for the minimum cost and the satisfaction of all the design constraints. This approach has been implemented into a KBE application connected with a commercial optimization package and tested on several routing cases. The results demonstrate that the proposed method is capable of handling cases of representative geometric complexity and design constraints and delivering proper 3D harness models in full automation.

Keywords EWIS - Wire harness - 3D routing automation · $\mathrm{KBE} \cdot \mathrm{MDO}$

\section{Design challenges in the development of the aircraft Electrical Wiring Interconnection Systems}

The Electrical Wiring Interconnection System (EWIS) is a very complex system due to its sheer size and the multitude of the interconnection requirements of on-board avionics. The EWIS of the A380 (Fig. 1), for example, contains $530 \mathrm{~km}$ of cables, 100,000 wires and 40,300 connectors 
[2]. Although the wiring volume tends to reduce thanks to the implementation of data-buses (e.g., the Boeing 737NG has several kilometres less wiring compared to its predecessor), the complexity of the EWIS is deemed to further increase in new generation More-Electrical-Aircraft (MEA) and Full-Electrical-Aircraft (FEA) [3]. Significant advances in the design method of such a system are necessary, not only to efficiently address the growing amount of electronic systems to be interconnected, but also to comply with the growing amount of safety regulations stipulated by Certification Authorities concerning reliability and redundancy [4].

The EWIS design process (see flow chart in Fig. 2), consists of two main parts, generally addressed as electrical design and physical design. In the electrical design part, the power and data signal interconnection between the various electronic components is defined; the wires and cables are selected according to the connection requirements, the estimated harness length and voltage drop. In the physical design part the actual routes of the many wire harnesses are developed. The physical design can be further divided into three phases, namely Space Reservation, Main Routing Architecture (MRA) design and 3D Routing.

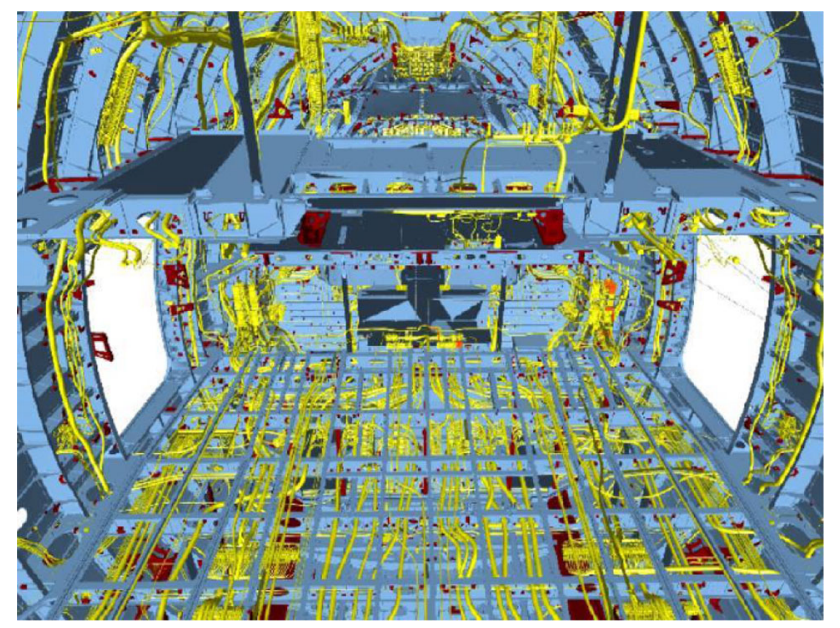

Fig. 1 Part of the Airbus A380 EWIS [1]
During the Space Reservation phase, space is reserved in the aircraft to allow the routing and integration of the EWIS. In this phase, EWIS designers and other designers responsible for the integration of other systems (e.g., air conditioning and anti-icing) compete for the scarce space available on the aircraft. The outcome of the MRA design is the definition of the so-called motorway network that designates the preliminarily routes for connecting the main aircraft systems. In the 3D routing phase the actual harness models are generated inside the aircraft Digital Mock-up (DMU), making use of the reserved space and defined MRA, to connect the various systems according to the electrical definition established in the electrical design part.

Within the physical design part, 3D routing is the most time consuming phase and requires the highest amount of engineering staff. This is caused by lengthy and repetitive work, mostly manual although partially supported by Computer Aid Design (CAD) systems, required to address the sheer amount of design requirements (e.g., no go areas, areas requiring special cables protections, allowable bend radius of cables, allowed structure to clamp the harness, etc.), and to deal with the frequent changes in the aircraft structure that might force, for example, to reconsider the position of some attachment points, or even define entirely new cabling routes because of reduced space availability. Due to the limited time allocated to perform their work and the frequent last minute changes, wire harness designers work under high pressure and their work is prone to errors. The methodology proposed in this paper addresses specifically this critical phase in the physical design part.

Considering the fact that a large amount of harness components are selected from catalogues and that the nature of the wire harness design work is largely repetitive and mostly rule based, there are lot of opportunities to automate the design process, thus releasing design engineers from lengthy and repetitive work and potentially increasing their creativity. Surprisingly, automating the harness physical design has not received much academic attention in spite of the technical challenges and associated improvement opportunities. A number of researchers [5-9]
Fig. 2 Overview of the EIWS design (bottom) in the overall aircraft development process (top)

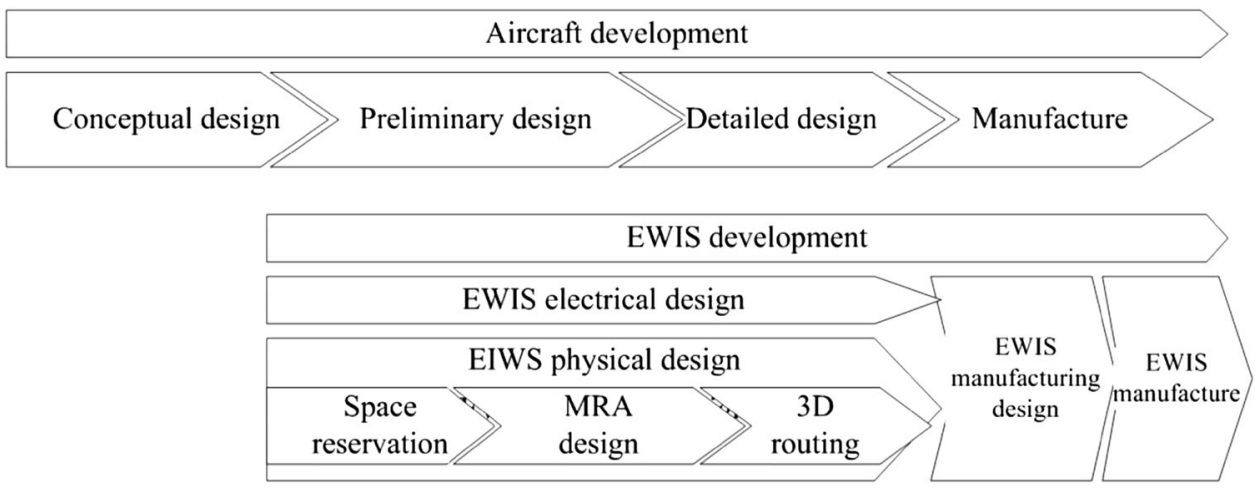


have focused on the automation of EWIS design. However, none or very limited solutions to automate the 3D routing part of the EWIS design process have been found. Also the current leading Mechanical CAD (MCAD) tools used in industry are not able to generate wire harness 3D models automatically and still demand a lot of manual work by expert designers.

\section{Knowledge Based Engineering and Multidisciplinary Design Optimization to automate 3D routing of wire harnesses}

This paper proposes a novel methodology to address the $3 \mathrm{D}$ routing challenges discussed in Sect. 1. In particular the proposed methodology aims at the following two main objectives:

1. Automate the generation of the $3 \mathrm{D}$ wire harness models, by capturing and systematically reusing the experts' knowledge;

2. Automatically update wire harness models when changes occur, either in the routing environment, or in the electrical design of the EWIS.

The proposed approach is based on the hypothesis that solving the $3 \mathrm{D}$ routing problem is equivalent to solving an optimization problem. In this case, the objective function to minimize represents the cost function that accounts for both the total cost of the wire harness and the cost of the protection layers and support components required to route the harness in area with harsh environment (heat, vibration, etc.). The design variables represent the position of the various clamping points where the cables are fixed to the aircraft structure and which, in practice, are used by the design engineers to control the position and shape of harnesses. The various design rules, such as minimum allowed bend radius, maximum distance between contiguous clamping points, and minimum distance between cable and support structure, are formulated as constraint functions for the optimization problem.

The technical implementation of the proposed approach is built on a combination of Knowledge Based Engineering (KBE) [10] and Multidisciplinary Design Optimization (MDO) [11] technologies. KBE technology is exploited to capture the typical rule-based approach of wire harness design and to enable the automation of all the geometric manipulations and checks required to perform the routing task. MDO is used to systematically explore the large design space provided by the $3 \mathrm{D}$ routing problem, to discover the minimum cost solution that complies with the multitude of design constraints.

Section 3 of this paper provides an overview of the EWIS architecture and the main design rules involved in wire harness routing. Sections 4, 5 and 6 describe the actual definition of the optimization problem and its technical implementation. In Sect. 7, examples are provided to demonstrate the capability of the proposed approach to meet the two objectives. Conclusions and recommendations are given in Sect. 8 .

\section{Description of aircraft EWIS and 3D routing rules}

Section 3.1 provides a description of the aircraft EWIS and clarifies the focus area of the proposed methodology. Section 3.2 details the design rules and checks to be applied during the $3 \mathrm{D}$ routing. Both subsections introduce the terminology that is then used in the formulation of the optimization problem.

\subsection{Description of the aircraft EIWS}

The aircraft EWIS propagates through almost every part of the airframe and the engines. To facilitate manufacturing and installation, it is designed as a set of separate harnesses, which, during assembly, are connected at the so called production-break points. The way the EWIS is split into different sets of harnesses strongly depends on the aircraft zones where the EWIS is routed. These zones are called wiring zones and differ from each other because of their environmental conditions, such as heat, vibration, and moisture. As consequence, each wiring zone may demand some different design rules. In practice, each wiring zone is independent from the others for what concerns the design and installation process. The earlier mentioned productionbreaks represent the only interface between the adjacent zones. The production-breaks are predefined and generally not modified during the $3 \mathrm{D}$ routing process.

Each wiring zone includes one or more harnesses, which connect the production-breaks and the pieces of equipment installed in the given zone. Each wire harness is generally divided further into branches, components, and sub-components, as illustrated in the EWIS hierarchy structure shown in Fig. 3 and the two annotated examples in Fig. 4. A wire harness can contain one or more branches; each branch contains one bundle, and may include one or two connectors, protection layers, and some clamps; each bundle contains one or more wires, where electrical or data signals are transmitted. The points where more branches converge on a wire harness are called breakout points. When breakouts are present, it means the given harness has multiple origins and/or multiple destinations. As a consequence, a branch is defined as a part of a harness that locates between two breakouts or a breakout and a connector (including the connector). 


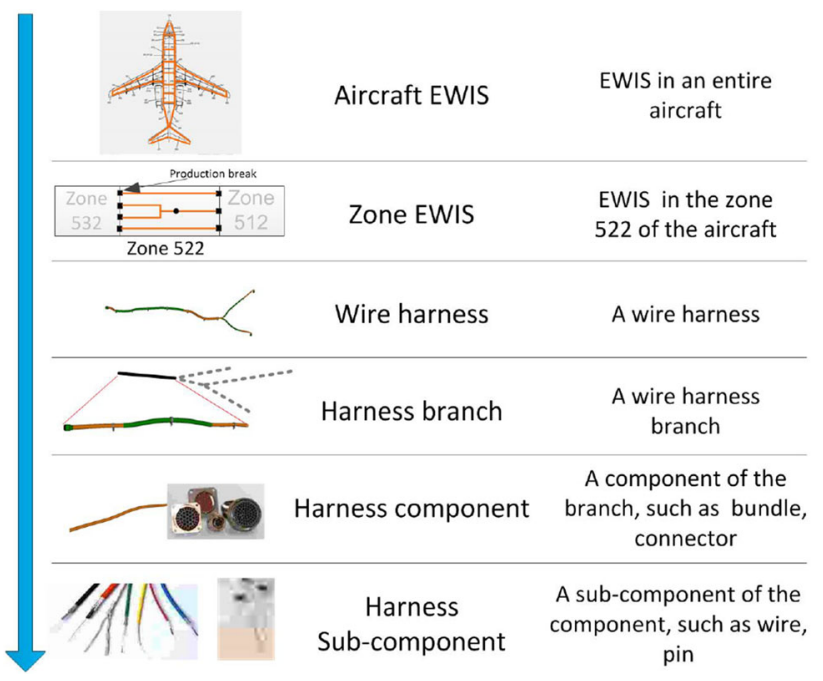

Fig. 3 Hierarchical structure of the EWIS

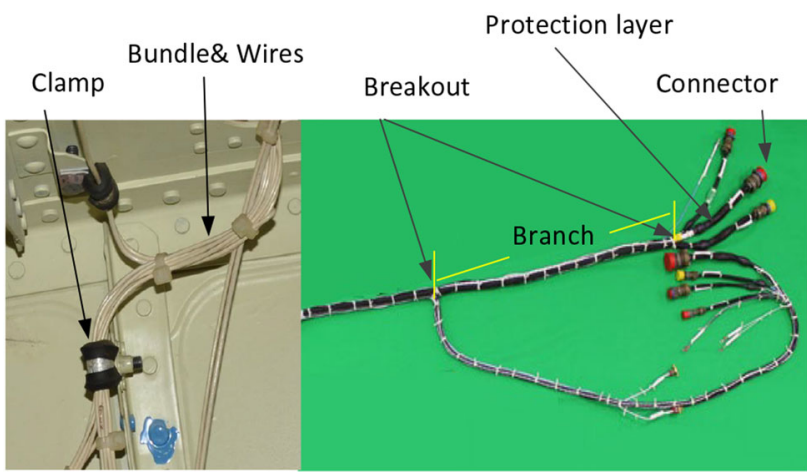

Fig. 4 Examples of wire harnesses and their main

\subsection{D routing rules}

The $3 \mathrm{D}$ routing process is constrained by many design rules. Some of these design rules are described in the design specifications issued by the authorities to guarantee the safety of the aircraft; others have been developed by wire harness manufactures themselves, on the basis of experience and best practice. A subset of these rules has been selected and implemented in this work. These include, for example, the rules to check allowed bending radii and geometry collision, as well as those for the definition of the clamping system and routing in critical wiring zones, such as in presence of heat sources or high flammability risk. All these rules are discussed in some detail in the following sub-sections.

It is important to note that the selected rule subset has been selected in consultancy with EWIS manufactures and consists of the most relevant ones, both in terms of the frequency of application and complexity of implementation. This subset was deemed sufficient to demonstrate the capability of the proposed approach, which, anyhow, was developed to guarantee full scalability, as further discussed in Sect. 6.

\subsubsection{Minimum bend radius rule}

A wire bundle or a cable must not be bent beyond its allowed limits to avoid damage. The minimum allowed bend radius of a wire bundle is determined by the product of its diameter and allowed bend radius ratio, namely $\varsigma \times D^{\text {bundle }}$. $D^{\text {bundle }}$ is the bundle diameter and $\varsigma$ is the allowed bend radius ratio, which mainly depends on the bundle material. The principle to select the bend radius ratio can be found in design specifications MIL-W-5088L [12] and Aircraft EWIS Best Practices [13]. The bend radius violation free of a bundle is represented by the inequality $r_{\min }^{\text {bend }} \geq \varsigma \times D^{\text {bundle }}$, where $r_{\min }^{\text {bend }}$ is the minimum bend radius of the bundle measured at the bundle centre curve. As mentioned in Sect. 2, KBE systems as the one used in this work allow performing this kind of geometrical check in a very efficient manner.

\subsubsection{Geometric collision rules}

When routing wire harnesses inside a wiring zone, it is necessary to check for three types of collisions in the geometry model: (1) collision between a harness and aircraft components, (2) collision between different branches of the same harness, and (3) collision between the harness being routed and any previously generated harness. Examples are illustrated in Fig. 5. None of these types of collisions is allowed. Similarly to the rule discussed in the previous section, KBE systems provide efficient methods to check for collisions among geometrical objects.

\subsubsection{Geometric attraction rule}

During 3D routing, harnesses have to be fixed to the airframe by means of some clamping device (from here on addressed as clamp). Not all airframe components are suitable or allowed to be clamped. For example, wire harnesses are not allowed to be fixed on aircraft systems.

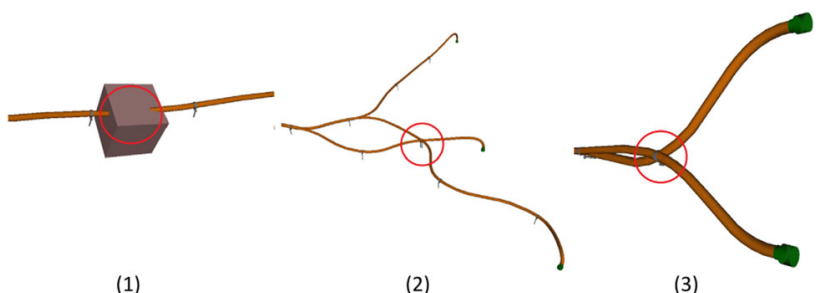

Fig. 5 Three types of geometric collision: (1) between harness and geometric structure (2) between branches of the same harness, (3) between harnesses in the same wiring zone 
Rules are necessary to guarantee only fixable structures (i.e., the structure that allows harnesses to be fixed on) are used for clamping. To minimize space occupation, as well as the weight of the clamps, it is convenient to route harnesses in proximity of fixable structures. This is the socalled geometric attraction rule. Also in this case, KBE systems provide the necessary geometry analysis and manipulation methods to support the implementation of this rule.

\subsubsection{Clamping and fixing distance rules}

Wire harnesses need to be fixed on the fixable structure at proper values of fixing and clamping distance (Fig. 6). The fixing distance is the distance between the harness centre curve and its fixing structure, measured in correspondence of the clamp. Due to the natural deflection, a harness bundle always sags between two clamps. As a matter of fact, minimum sagging values are necessary to avoid harnesses undergo tension when the fixing structure deforms under external loads. The fixing distance must be sufficiently large to avoid any contact (hence abrasion) between the sagging harness and the structure. On the other hand, it should not be too large to limit the size (thus cost and weight) of clamps, stand-off, and brackets, as well as to minimize the space occupied by the EWIS. Inside the fuselage, for example, the normal sagging value is $1 / 2$ inch, measured on the bundle centre curve, half way in between two contiguous clamps. Therefore, the minimum fixing distance necessary to avoid chaffing and abrasion can be calculated by the Eq. (1). In practice, the actual fixing distance is always larger than this value to guarantee some margin.

min fixing distance $=1 / 2$ inch + harness radius

The clamping distance is the distance between two adjacent clamping points, which include, besides the actual clamps, also connectors and breakouts. The maximum allowed clamping distance depends on the harness material and the routing environment. For a normal harness routed in a no-vibration zone (e.g., in the fuselage cabin) this distance is 24 inches. For rigid harnesses this distance is extended to 42 inches [12]. Inside wings, in correspondence of engine mounts, the clamping distance needs to be smaller to handle the vibration caused by the engines.

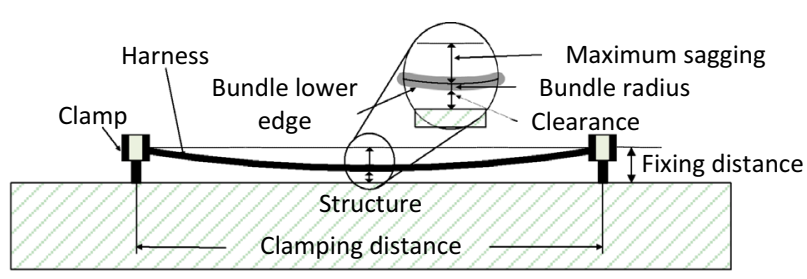

Fig. 6 Definition of clamping and fixing distance

\subsubsection{Grey areas rules}

In an aircraft, there are lots of hazardous zones, such as wet, hot and vibratory areas. These zones are neither forbidden (i.e., black) nor free (i.e., white) for harnesses to be routed, thus addressed here as grey areas. By employing special precautions such as protection covers and extra clamps, harness can be routed in grey areas. In the work presented here, some routing rules have been implemented, now limited to the cases of hot and flammable zones, which influence the use of protection cover and clamps, respectively. Extra rules of similar nature, e.g., for wet or corrosive areas, can be easily added.

3.2.5.1 Rule for hot zones Hot zones are common areas in an aircraft. They are located around high-temperature equipment such as resistors, exhaust stacks, and heating ducts. Harnesses exposed to high temperature suffer deterioration and deformation. Therefore, it is necessary to 'insulate wires that must run through hot areas with a hightemperature insulation material.' [12]. The extra cost incurred by the use of dedicated protection can be avoided by routing the harness outside these hot zones if applicable, at the cost of a longer harness. It is a trade-off process for designers to find the most cost efficient solution. In the approach presented here, it is responsibility of the optimizer.

3.2.5.2 Rule for flammable zones Areas in the surrounding of flammable fluids or gas pipes are examples of typical flammable zones. An arcing fault caused by broken wires in this area may result in a fire. For wire bundles routed above fluid lines, the design specifications demand that "the clamps should be of compression type and should be spaced so that, assuming a wire break, the broken wire will not contact hydraulic lines, oxygen lines, pneumatic lines, or other equipments whose subsequent failure caused by arcing could cause further damage." [13] The larger amount of clamps that is necessary to route in these zones affects the total cost and weight of a harness. This extra clamping cost can be avoided by routing the harness outside flammable zones, at the cost of a longer harness. Similarly to the earlier mentioned case of hot zone, it is also a trade-off process for designers, or for the optimizer in our case, to find the best solution.

\section{Design methodology for automatic 3D routing}

As anticipated in Sect. 2, the method proposed in this paper to automate wire harness $3 \mathrm{D}$ routing is based on the hypothesis that a routing problem can be solved as an optimization problem. The optimization problem definition for the 3D routing is presented in Eq. (2). 


$$
\begin{aligned}
& \underset{x}{\min } f(x) \\
& f(x)=\sum_{j=1}^{m} f_{i}(x)=\sum_{j=1}^{m} L_{j}(x) C o_{j}(x)
\end{aligned}
$$

with respect to $x$

$$
\text { subject to } C_{i}(x) \leq 0
$$

The objective function is the harness cost function (e.g., in Euros), which is the summation of each branch cost $f_{j}(x)$ defined as the product of the harness branch length $L_{j}$ and branch cost coefficient $\mathrm{Co}_{j}$, namely the unit length cost of the given branch. The cost coefficient $C o_{j}$ is the summation of the following three sub-cost coefficients:

- Cob sub-cost coefficient of bundles. This depends on the diameter of the bundle;

- Coc sub-cost coefficient of clamps. This depends on the amount of clamps used to fix the given branch to the airframe, where the amount of clamps results from the application of the clamping distance and flammable zone rules;

- Cop sub-cost coefficient of protections. This depends on the amount and type of protecting layers used when routing the given harness branch through hot areas (hot zone rule application).

The cost in this object function includes the material cost and a part of the installation cost (included in the clamping cost). In practice, this function can include more cost items, such as the manufacturing cost and a part of the operation cost (i.e., fuel consumption caused by the harness weight), using the cost calculation methods provided by harness manufacturers.

The design variables $x$ represent the coordinates of the clamps and breakout points of a wire harness (both indicated with dots in Fig. 7). In the current manual design process, clamps and breakout points are actually used by EWIS engineers as way-points to control the position and shape of harnesses inside the aircraft Digital Mock-up (DMU). In the automatic 3D routing approach proposed here, it is the optimizer that moves these points ${ }^{1}$ to systematically explore the entire routing space.

The optimization parameters (i.e., the parameters that do not vary during the optimization) include:

\footnotetext{
$\overline{1}$ In practice, both the position and the direction of clamps and breakouts are used to define harnesses. In the current implementation of the optimization process for automatic 3D routing, however, only the positions are used as design variables, whilst the directions are assumed to be function of the clamping point positions and the design environment. The adoption of directions as extra design variables would be possible at the cost of extra computational time, without significant changes to the implementation of the proposed approach.
}

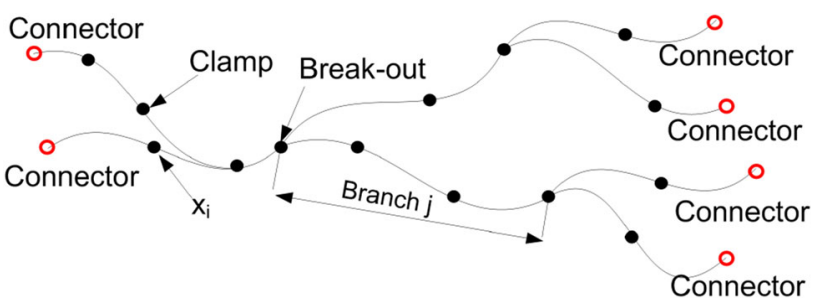

Fig. 7 Wire harness representation as used for the routing optimization

1. The position of the production breaks and various electrical systems receptacles, i.e., the origins and destinations of the harness to be routed.

2. The bundle diameters, which are determined by the number and gauge of the wires present in the given bundle.

3. The routing environment geometry, including information, such as location and temperature of eventual hot zones.

4. All the coefficients used by the various design rules, such as the allowed clamping distances and bend radii, and the cost per unit/length of cables, covering material, clamps, etc.

The optimization constraints $C_{i}(x)$ are formulated on the basis of the earlier discussed design rules. For example, $C_{\text {bend }}(x)=\varsigma \times D^{\text {bundle }}-r_{\min }^{\text {bend }}$ and the bend radius rule will be satisfied if $C_{\text {bend }}(x) \leq 0$. As discussed later in detail, some constraints are actually included in the objective function as penalties, rather than being formalized as inequalities.

\subsection{Two-steps optimization strategy}

The challenge to solve the optimization problem described above is that the number and initial value of the design variables are not known a priori. Indeed, the number and position of clamping points are output of the harness routing process. To handle this 'chicken-and-egg' type of problem, a two-step, hybrid optimization strategy has been devised. In the first step, called Initialization, a grid of potential clamping points is generated in front of each of the structural elements where the wire harnesses are allowed to attach. Then an optimization approach is applied to route a simplified harness model on such grids. As a result of this initialization step, a preliminary routing of the harness is obtained, together with the number and position of its way-points (i.e., the number of clamp and breakout points and their coordinates). Once the number of design variables and their initial values are known, the second optimization step can be applied. In this second step, called Refinement, the actual geometric model of the wire harness is generated based on the way-points from the 
Initialization, which are here varied by another optimizer to minimize the harness cost objective function, while satisfying all the design constraints. These two steps will be elaborated in Sects. 5 and 6, respectively.

\subsection{KBE framework for hybrid optimization}

KBE has been used to support the two-step optimization process. A KBE application was developed using a commercial KBE system, to take care of all the geometric modelling and querying operations necessary 1) to compute the state variables and objective function and 2) to evaluate the constraints function during the various optimization loops. In particular, the KBE application is taking care of reading in the geometry of the aircraft (i.e., the routing environment), generating the grids (see details in Sect. 5.2) for the Initialization phase modelling the harnesses as lofted surfaces along b-splines curves, measuring harness data such as bundle lengths and bend radii, and checking for the violation of design rule such as geometric collisions. The actual optimizations are performed by a separate commercial optimization toolbox, linked to the KBE application.

The resulting computational framework (KBE app plus optimizers), called Harness Design and Engineering Engine (HDEE), was architected according to the DEE concept described in [14-16]. Figure 8 illustrates the four main components of the HDEE with their connections, namely the Initiator, the Optimizer, the Multi Model Generator (MMG) and the Analysis tools. The Initiator is responsible for generating the preliminary harness definition. The Optimizer is in the charge of the systematic exploration of the routing space and convergence checks. The MMG is the component responsible for the generation of the harness geometric model and the extraction from such model of the specific data required for the analysis tools. These analysis tools include modules to calculate the cost of the harness and check whether the harness configuration satisfies the design rules, such as bend radius violation and geometric collision. The first of the two optimization steps, the Initialization, is performed by the Initiator block and is discussed in Sect. 5. The other three blocks are used in the Refinement step, which is described in Sect. 6.

\section{D routing initialization}

The Initialization step is responsible for generating a preliminary harness definition, which is then used as the starting point for the Refinement. In the initialization step, a road map-based optimization method is adopted. Similar to

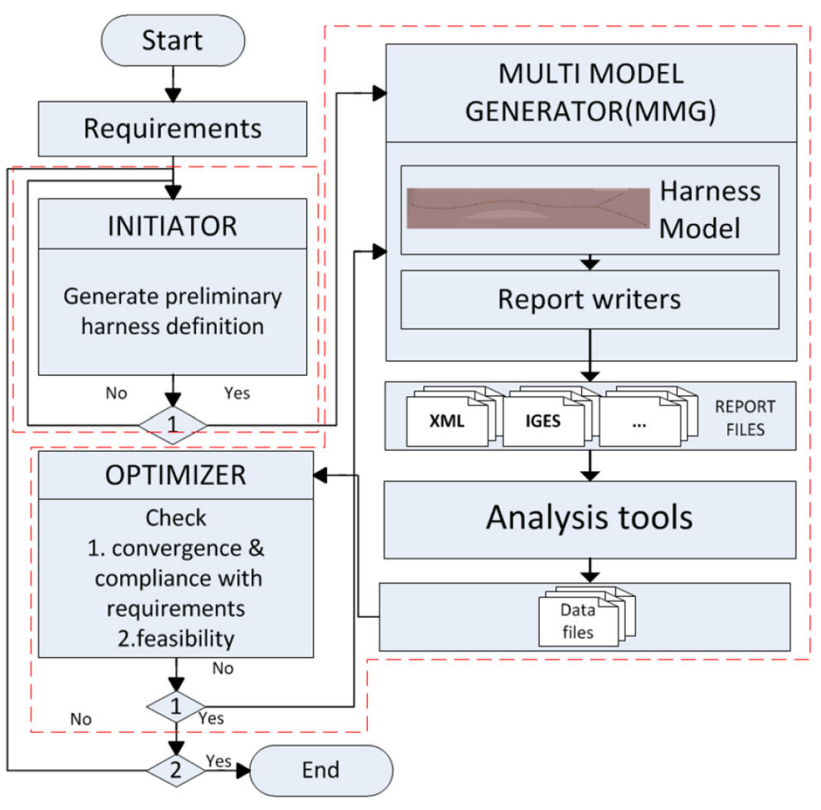

Fig. 8 Harness Design and Engineering Engine (HDEE)

a conventional GPS navigation system or a path finding process for computer games [17, 18], this optimization method first builds a road map (i.e., a grid of nodes and connections) in the routing environment and then uses it to find a convenient path for the harnesses. Typical path finding methods/algorithms, such as those implemented in GPS route planners, however, are able to find only paths that have one origin and one destination. These methods cannot solve the path finding problem for wire harnesses that have multiple origins and destinations (from here on simply addressed as multi-destination), as the one schematized in Fig. 7. To handle the multi-destination feature, a bi-level optimization strategy is proposed.

\subsection{Bi-level optimization strategy}

The bi-level optimization decomposes the path finding of an entire harness into the harness (global) level and the branch (local) level, as illustrated in Fig. 9. These levels are coordinated by the breakout points as follows: the global level optimization moves the breakout points to different nodes on the road map to explore the routing space. Meanwhile at the local level, several road map based optimizations search for the shortest paths between adjacent breakouts (or between breakout and receptacle) and return the cost of each branch paths to the global optimizer, who adds them and, accordingly, modifies the breakout point positions for the next iteration. This process iterates until the harness configuration is converged. The generation of the road map and the global and local optimization processes are detailed in the next three subsections. 
Fig. 9 Architecture of the bilevel path finding method

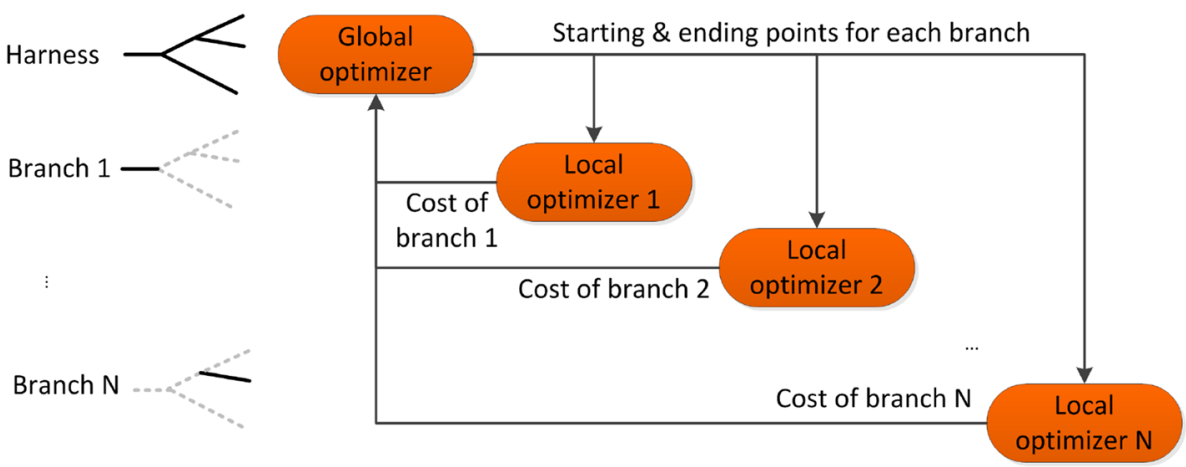

tessellation size, i.e., the distance between adjacent nodes in the tessellation, is calculated on the basis of the clamping distance discussed in Sect. 3.2.3. Thus, different areas can get different tessellation density.

- 3 roadmap generation In the fourth and final step, the nodes on the adjacent tessellated surfaces are first connected to generate one continuous 3D road map in front of the fixable structure. Then, the geometric collision and clamping distance checks are performed for all the edges of the map (i.e., the sides of all the tessellation shapes). Any edge colliding with any geometric component or longer than the allowed clamping distance is excluded from the final 3D road map. Edges crossing grey areas get a specific coefficient assigned to account for the extra cost, later during routing. Edges locating in EWIS reserved areas get another coefficient (negative cost) to "invite" the routing algorithm to pass through them.

Once the road map is generated (see example in Fig. 11), it is possible to proceed with the preliminary 3D routing. Each node on the map can be used as potential
Fig. 10 The 4 steps to generate the harness road map (1) geometry and environmental zone data read in (2) Offset; (3) Tessellation; (4) Roadmap generation

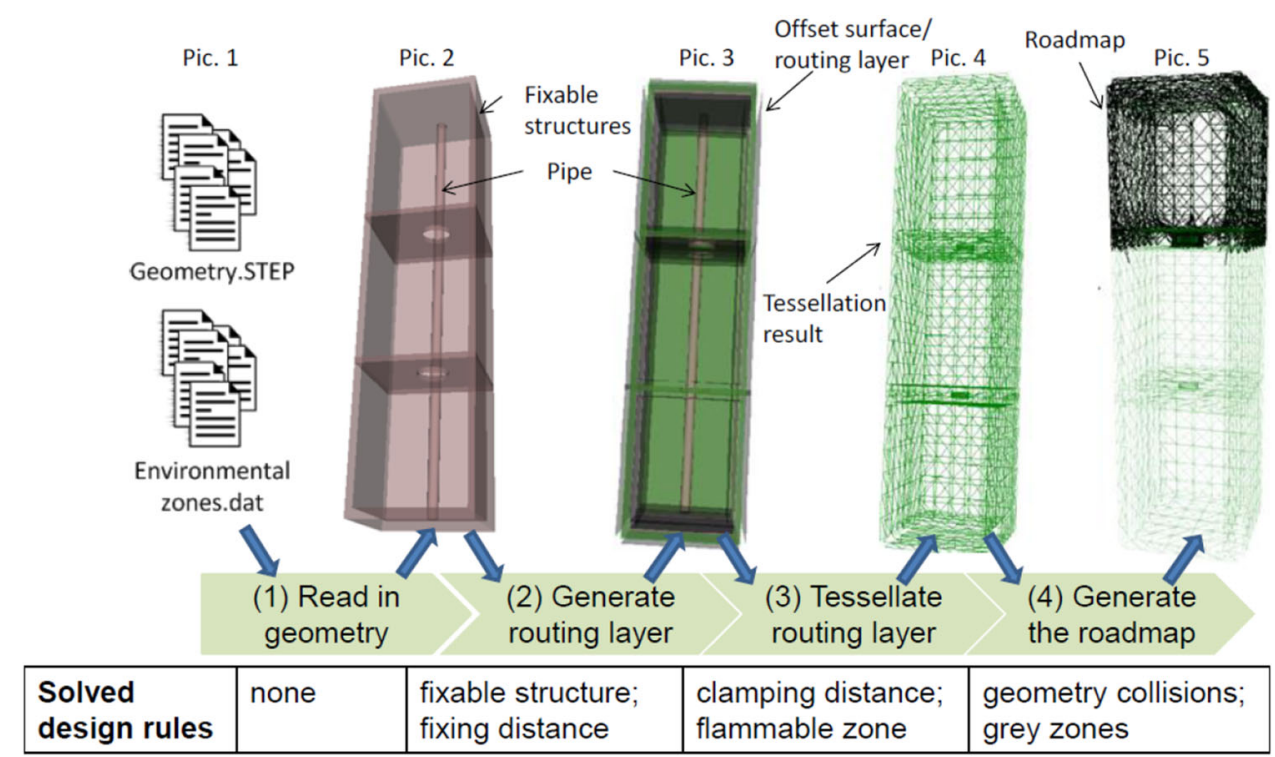




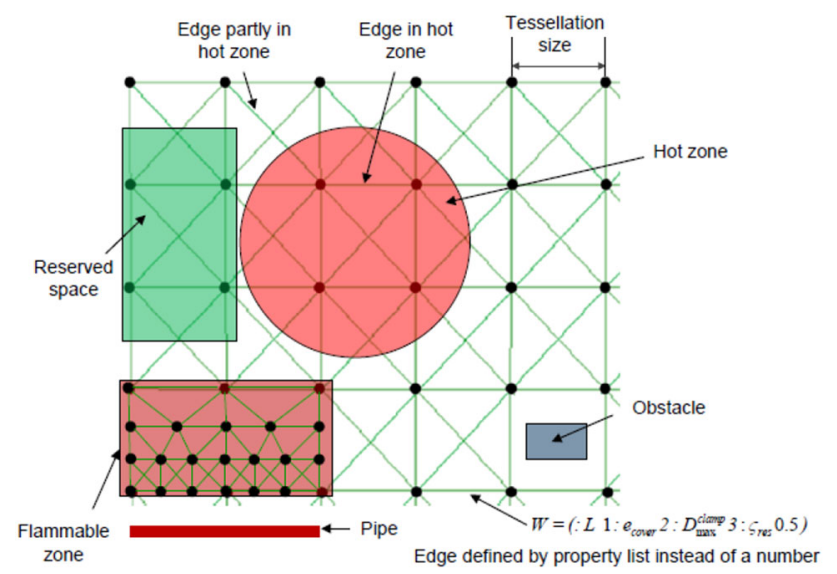

Fig. 11 Road map of a typical routing environment

clamping point, and any wire harness branch can be defined as a polyline built by chaining contiguous edges in the road map.

A check is performed to determine whether some of the road map edges locate in harsh environment areas, where, for example, protection covers would be required to protect the harnesses, as discussed in Sect. 3.2.4. In case this happens, specific cost coefficients are assigned to those edges to account for the penalty of routing in that area during the optimization.

\subsection{Local level optimization}

A modified A* algorithm [19] is adopted to handle the local level optimization. Any $\mathrm{A}^{*}$ optimization algorithm is able to find the optimum path between two given points, addressed as source node and destination node, without knowing a priori the number of design variables. The special $A^{*}$ algorithm used in this research differs from other $\mathrm{A}^{*}$ algorithms in the aspects discussed in the two following subsections.

\subsubsection{Cost calculation method}

The implemented $\mathrm{A}^{*}$ algorithm uses the cost function $f(n)=g(n)+h(n)$ to evaluate the cost of the traversed nodes along any given path. Each of the nodes is addressed as $n$ when it is evaluated. The function $g(n)$ is the movement cost from the source node to the current node $n$. The function $h(n)$ is an estimated movement cost from current node $n$ to the destination node. The movement cost along a map edge is generally represented only by the length of the edge. Here, the movement cost along an edge is the product of the edge length by the cost coefficient assigned to that edge during the 3D map generation. The formula used for the movement cost calculations is shown in Eq. (3). It should be noted how this cost function does not only depend on the amount and type of material, but also on manufacturing and installation aspects, such as the extra costs for manufacturing harnesses with special protection and the installation of the clamps, respectively.

$$
\begin{aligned}
& f\left(r_{\text {bundle }}, p_{u}, e_{\text {bundle }}, C_{m}, C_{i}, L, D_{\text {max }}^{\text {clamp }}, e_{\text {cover }}, t h_{\text {cover }}\right) \\
& =L C o=L(\text { Cob }+\operatorname{Coc}+\operatorname{Cop}) \\
& =L\left(\pi r_{\text {bundle }}^{2} p_{u} e_{\text {bundle }}+\frac{\left(C_{m j}^{k}+C_{i}\right)}{D_{\text {maxj }}^{\text {clamp }}}\right. \\
& \left.\quad+\pi p_{u} e_{\text {cover }}\left(2 r_{1}^{\text {cover }} t h_{\text {cover }}+t h_{\text {cover }}^{2}\right)\right)
\end{aligned}
$$

$L$ length of the road map edge, $r_{\text {bundle }}$ radius of the bundle cross section, input, $p_{u}$ unit price, input, $e_{\text {bundle }}$ density of the bundle material, input, $C_{m}$ material cost of the clamp, depends on $r_{\text {bundle }}, C_{i}$ clamp installation cost, input, $D_{\max }^{\text {clam }}$ maximum allowed clamping distance, depends on the routing environment and is one of the attributes assigned to the roadmap edges, $e_{\text {cover }}$ density of covering material, depends on the routing environment and is an attributes of the roadmap edges, $t h_{\text {cover }}$ thickness of covering, input, $r_{1}^{\text {cover }}$ inner radius of covering, the same as $r_{\text {bundle }}$.

The total branch cost is the summation of all edges cost. When calculating $g(n)$, the actual value of the parameters will be used. When calculating the heuristic value $h(n)$, the maximum clamping distance and cost coefficient of covering in the non-grey area will be used to keep the so-called Admissibility [19] (i.e., to make sure $A^{*}$ can find the optimum path) of the algorithm.

\subsubsection{Bend radius check}

During the implementation of the $\mathrm{A}^{*}$ algorithm, the Curve Segment Bend Radius Pre-Calculation (CSBPC) approach is used to check for harness bend radius violations. This approach uses the current node $n$, the previous adjacent node $n-1$ and the next adjacent node $n+1$ to generate a fitted curve (see Fig. 12). At the start and end points the approach uses the vectors of the two points to build two auxiliary nodes $n-1$ (for start point) and $n+1$ (for end point) respectively, since these two nodes do not exist. Then it uses the minimum radius of this 3-point fitted curve to represent the actual minimum bend radius of the harness centre curve to check,

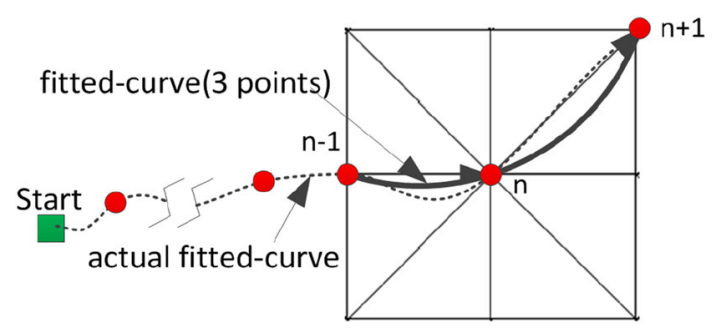

Fig. 12 Minimum bend radius check method in the initialization 
Fig. 13 Snapshots of the harness global pathfinding process during harness Initialization
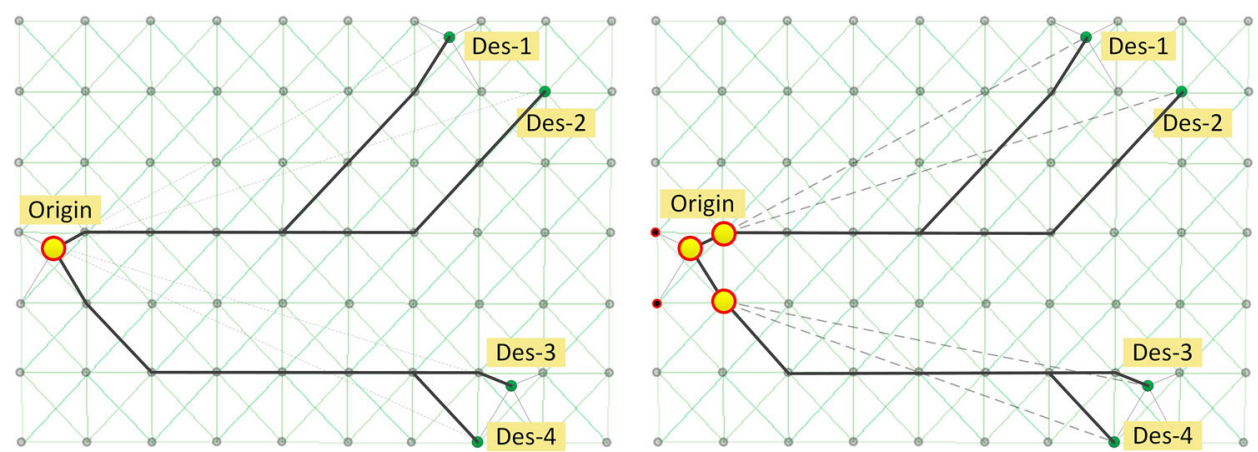

A

B
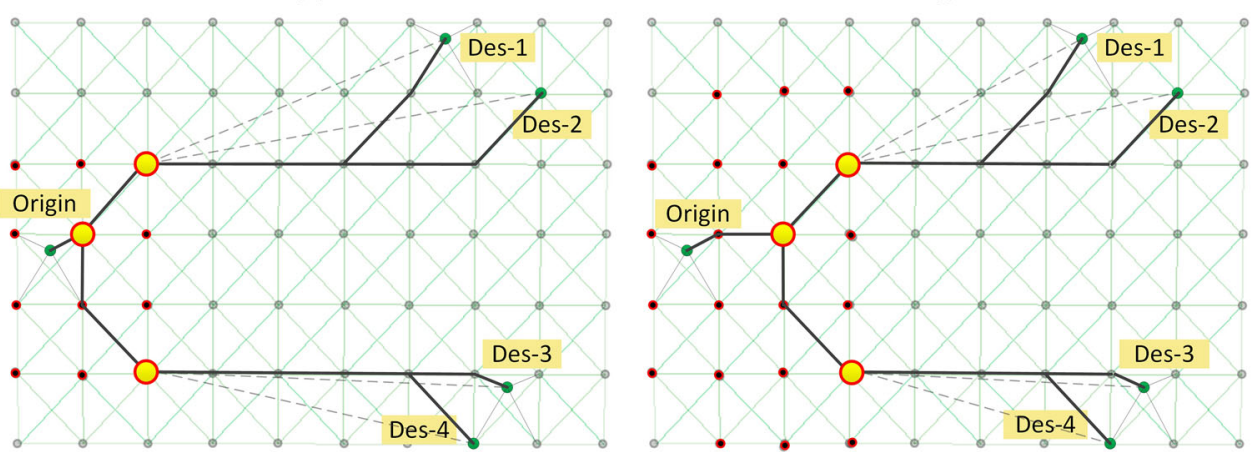

C

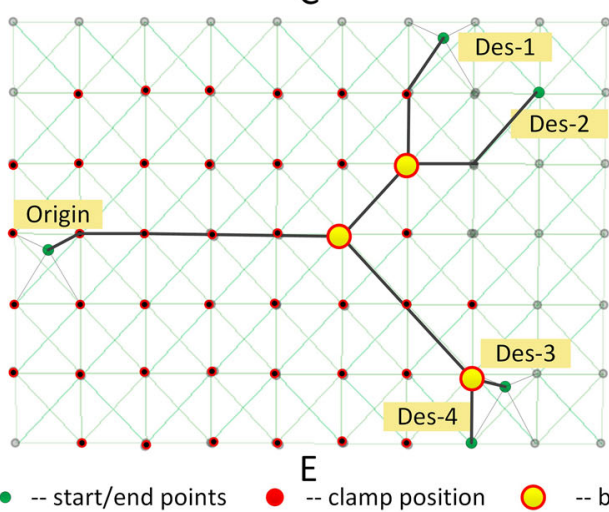

D

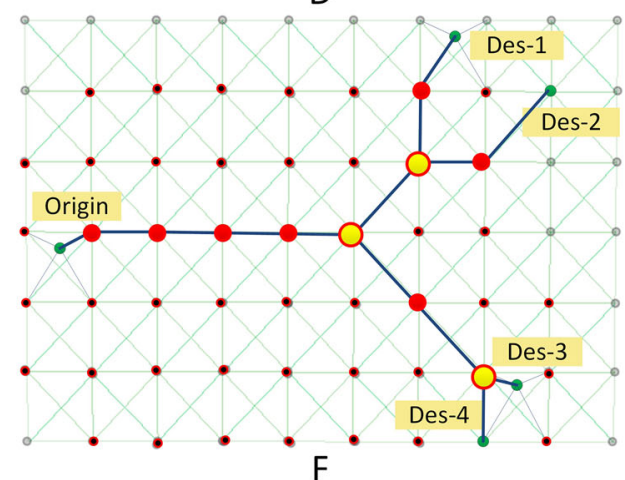

by means of a bend radius violation check function, whether the next node $n+1$ is suitable for the harness. The check starts from the start point and continues until the target point is found that has minimum cost on $f(n)$ and does not violate the minimum bend radius condition.

\subsection{Global level optimization}

The objective function for the global level optimization is defined as the summation of all branches cost. See Eq. (4).

$f(x)=\sum_{j=1}^{N_{b}} f_{j}(x)$

Here, $f_{j}$ is the cost of the harness' branch $j . N_{b}$ is the total number of branches and is determined during the electrical design and given as an input here. The vector $x$ consists of the design variables of the global level optimization, which are the positions of the harness breakouts.

The global level optimization uses the Hill Climbing algorithm [20, 21]. The algorithm moves each breakout to new locations on its adjacent nodes of the road map to get different breakout sets. The local path finding is carried out using these breakout sets. According to the local path finding results, the global optimizer evaluates the cost function and finds out the best breakout combination from the sets. If the best one is better than the benchmark in terms of objective function, this value will be set as the new benchmark and this design variables combination (i.e., this new collocation of the breakouts) will be accepted. The accepted design variables will be used as start position in the next loop. This process iterates until no further improvements can be found. An example of this process is shown in Fig. 13. 


\section{Harness 3D routing Refinement}

After the generation of the preliminary harness definition, the Refinement step starts with the coordinates of clamping points and breakout positions as design variables. The Refinement is in charge of refining the position of clamps and breakouts (but not their number!) to solve the limitations of the Initialization, namely, to eliminate the violations of some design rules and to optimize the preliminary harness routing even further.

\subsection{Limitations of the Initialization step}

The preliminary harness routing performed in the Initialization step by means of the road map has a number of limitations, which generally prevents achieving not only an optimal wire harness routing, but even a feasible solution.

\subsubsection{Inter-harness and inner-harness geometric collision}

A wiring zone may contain more harnesses. The path finding in the Initialization is implemented on each harness individually, without considering the presence of other harnesses. Hence, in some cases, different harnesses may share the same edge and/or vertex of the road map, which means geometric collision between different harnesses. Different branches of the same harness may also have a similar problem.

\subsubsection{Geometric collision between harnesses and geometric components}

In the Initialization, a wire harness is defined as a polyline chaining a number of edges of the road map. In reality, the wire harness is not a line but a cylindrical surface with a certain diameter. Its centre line is not a polyline but a spline curve fitting the nodes of the polyline generated by the Initialization. As a consequence, when generating the actual geometry of the wire harness starting from the polyline, even if this was geometric collision free in the Initialization, collisions between the actual harness and aircraft DMU components may actually occur (Fig. 14).

\subsubsection{Bend radius violation}

In the Initialization, the CSBPC method is applied to exclude some solutions having extreme bend radius violation. This method uses the minimum bend radii of curves generated using three adjacent nodes to carry the bend radius violation check on the whole harness. However, this method cannot guarantee these two minimum bend radii are the same. It also cannot guarantee a bend-radius-violation-free solution for an entire harness.

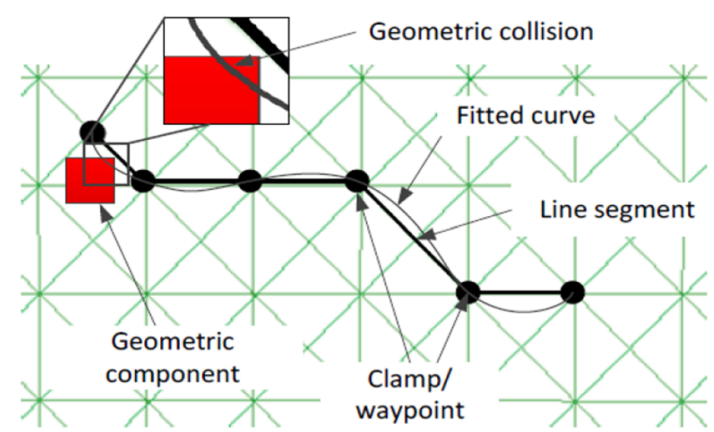

Fig. 14 Geometry collision of collision-free harness from the Initialization phase

\subsubsection{Non-optimum result}

In the Initialization, the bi-level optimization method finds the optimum harness within the limits set by the defined road map. Indeed the road map forces the harness to follow the edges of the fictitious map. Therefore, the Initialization is likely to produce a sub-optimal result in terms of the actual problem definition.

\subsection{Optimization approach in the Refinement step}

The limitations discussed in the previous section make the result of the Initialization only a preliminary harness definition. To finalize the harness path, a more conventional optimization approach is used to move the position of breakouts and clamping points (i.e., the design variables) freely in the routing space without using any road map anymore. The architecture of this optimization framework is illustrated in Fig. 15.

The initial values of the design variables $x_{0}$ are the Initialization outputs. These values are sent by the Initiator to the harness MMG. The harness MMG makes use of a predefined set of harness parametric modelling modules, called High Level Primitives (HLP), to build an actual geometric model of the wire harness Fig. 16.

Other specific modules in the MMG, called Capability Modules (CMs), extract from the harness geometric model the specific set of data $z_{i}$ required by the HDEE analysis tools. These analysis tools include modules to calculate the cost of the harness and to check the violation of the design rules. The results of these analysis tools, $f$ and $C_{i}$, are sent back to the optimizer to support the decision making for next iterations.

The Generalized Pattern Search (GPS) [22] algorithm is selected for the optimization. This algorithm, also known as black-box search, is gradient-free and is able to handle Boolean-output from analysis tools, such as the geometric collision check tool. This algorithm carefully moves the design variables to new positions to get new harness 
configurations. This feature is very useful since the Initialization already generates a promising harness definition. The algorithm moves the design variable $x_{i}$ one by one to $x_{i} \pm \Delta x$, to get new harness configurations. The time complexity of the algorithm on the number of design variables is $O(n)$. This feature keeps the calculation efficiency linear to the complexity of the harness, i.e., the number of clamps and breakouts.

Since the pattern search is not able to handle design constraints directly, these are implemented as penalties in the objective function. Thus, the objective function originally presented in Eq. (2) is modified as shown in Eq. (5). $\min \theta(x)$

$\theta(x)=f(x)+c_{\text {clamp }}(x)+c_{\text {bend }}(x)+c_{\text {geometry }}(x)$

with respect to $x$

The definition of the objective function $f(x)$ is the same as Eq. (3) except that the length $L$ here is the actual harness length (summation of all branch lengths) rather than the length of the map edge. The penalty value associated with each constraint violation is very high. Therefore, the optimizer will attempt to eliminate them first. An example of cost function $\theta(x)$ evolution during the optimization of a given harness is shown in Fig. 17. The preliminary harness configuration of this example had three constraint violations: two bend radius violations, and one geometric collision between two branches. With the progress of the optimization, the optimizer moves the design variables such to eliminate all the constraint violations first, because of the large benefits to the objective function. Indeed, the elimination of each constraint violation makes $\theta(x)$ drops steeply. As soon as the optimization reaches a feasible area, the constraints part of the objective function becomes null and $\theta(x)$ becomes the original objective function $f(x)$.

The optimization continues until the design has converged and/or the number of iterations has reached its set maximum (Fig. 17 bottom). At the end, the actual feasibility of the produced routing can be verified, inspecting the value of the various constraint functions, collected in the produced log file.

The $\mathrm{A}^{*}$ and the Hill Climbing algorithms used in the Initialization step, as well as the Generalized Pattern Search algorithm used in the Refinement, all generate the same output for a given input set. Thus, the overall results produced by the HDEE are deterministic. As far as the routing environment and the electrical system definition stay the same, the same 3D routing results are obtained.

It should be noted that, both the definition of the cost objective function, with its summation of penalty functions, and the modular architecture of the optimization framework (Fig. 15) should allow easy extensions of the proposed 3D routing approach with extra set of rules and constraints, e.g., to account for other type of grey areas, or other aspects related to installation and maintenance. For example an installation complexity factor could be added to account for small bending radii and inconvenient location of clamps. A maintenance factor could be established based on aspects such as number of clamps, number of cables per bundle, accessibility space. The approach would be the same: include extra geometry check modules exploiting the KBE system ability in dealing with geometry analysis and manipulation and add extra penalty functions to the objective function.
Fig. 15 Framework of harness design optimization

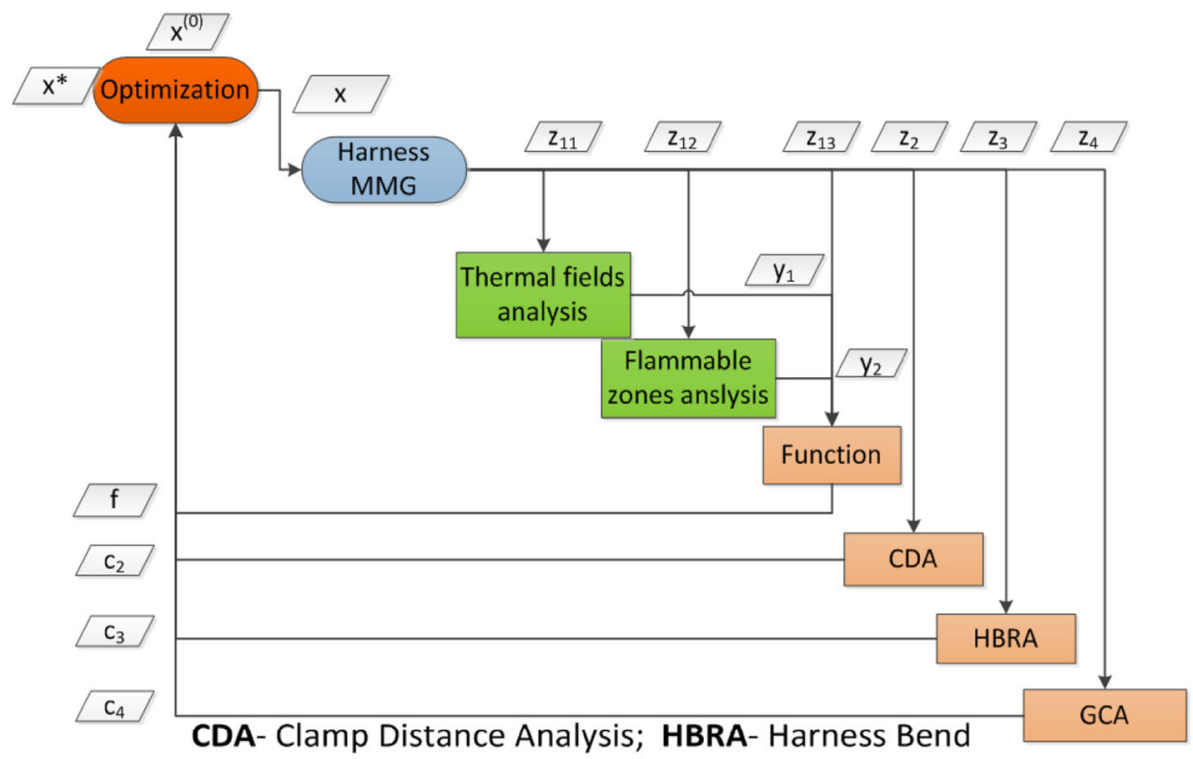

Radius Analysis; GCA - Geometry Collision Analysis 
Fig. 16 A limited set of High Level Primitives is used to model any wire harness bundle

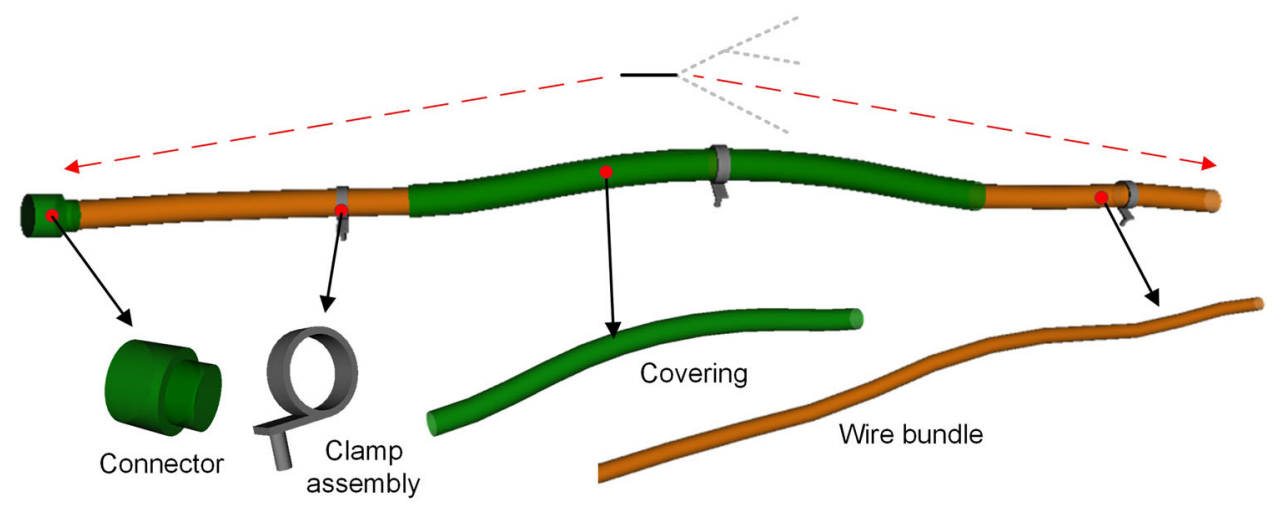

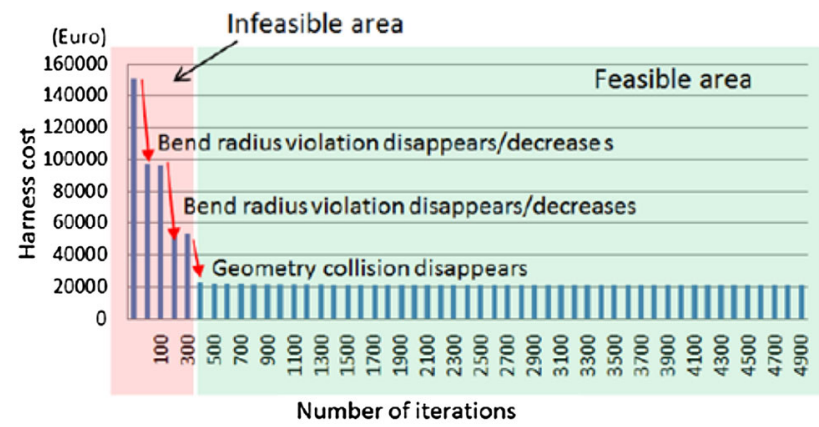

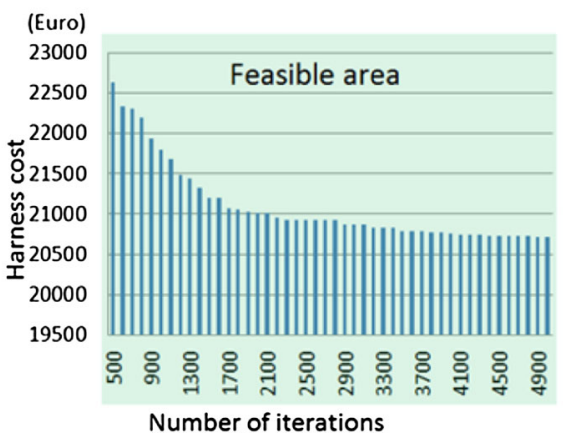

Fig. 17 Convergence history plot of the harness cost objective function (top). Detail of the convergence plot after resolution of all constraints violation (bottom)

\section{Results}

The proposed two-steps hybrid optimization approach has been implemented into the HDEE framework introduced in Sect. 4.2. Several test cases have been performed to validate the functionality of the application. The actual results of the routing process, i.e., the 3D models of the produced harnesses can be visualized immediately within the HDEE, and/or exported in the form of STEP files, and/or plain ASCII files (detailing the location and orientation of all the clamps and breakout points, as well as the diameters of all the branches). Using STEP files, the geometry model of the routed harness can be immediately transferred into the CAD system that was used to generate/deliver the DMU in the first place. Otherwise, some macros could be developed in the destination CAD system to automatically re-generate the geometry of the routed harness, based on ASCII files mentioned before. The export options provided by the HDEE are of paramount importance, because these options remove the need of a direct integration of the HDEE, or any of its capabilities, with the CAD system used by the EWIS manufacturer. As a consequence the HDEE capabilities remain intact when the version or even the type of CAD system changes per programs or manufacturer.

A few results produced by the HDEE are showed below here. Figure 18 shows routing results inside a fuselage barrel at the end of the Initialization and Refinement phase, respectively.

Figure 19 show the results of the routing process independently applied to two contiguous fuselage barrels, one including a geometrical obstacle in the cargo bay. The harness of the front and rear barrel connect at the production breaks. If the routing environment in one of the two barrel changes, the routing process can be re-executed only in that one, as far as the production breaks remain at the same location.

It should be noted that in case multiple harnesses need to be routed in the same environment, as in the previous example, the optimization process is performed one harness at time. Geometric collision between different harnesses is prevented by adding the previously routed harness to the list of the geometric components included in the routing environment. Since the order in which the harnesses are routed influences the final result, a sequencing rule has been implemented, based on EWIS manufacturers' best practice, that is, the thickest harness is always routed first. In real practice this is generally done because the thickest is also the most rigid and difficult to rework, but this approach does not guarantee to lead to a global optimum design. An interesting extension to the proposed approach would be that of routing all the harness belonging to a given area simultaneously. This would be possible at the expense of a much large computational cost.

Figure 20 shows the effect of a reserved space definition on the routing results. In both cases no constraint violations 

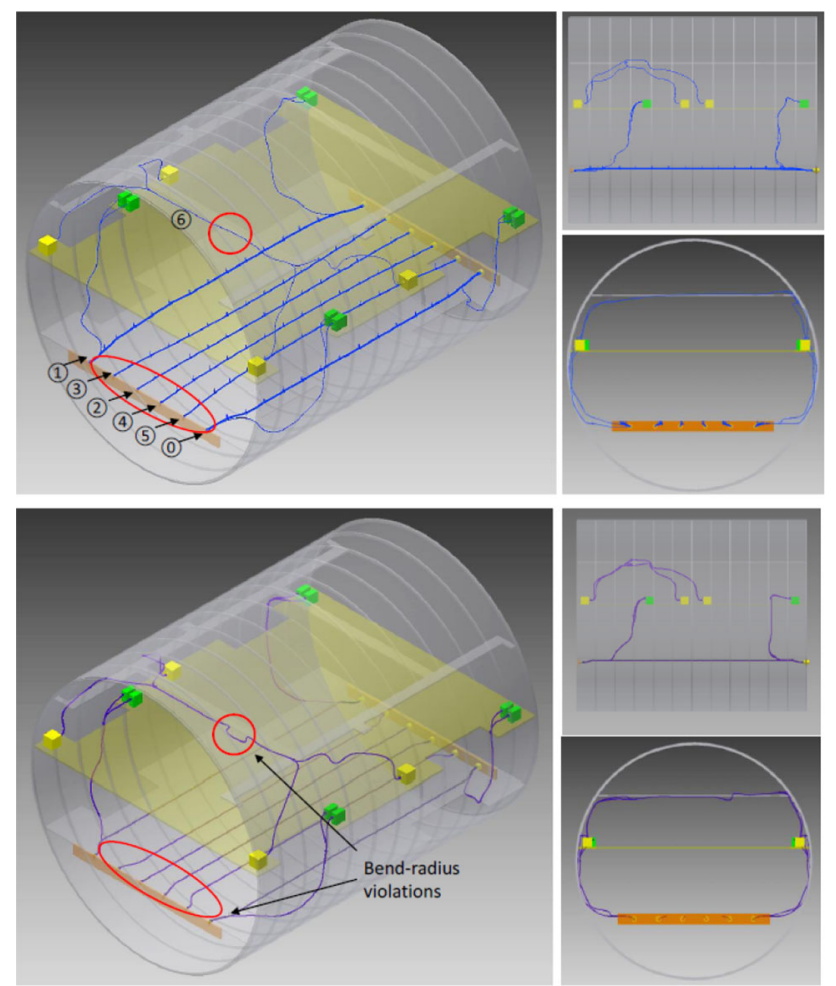

Fig. 18 Top: Initialization result of a wire harnesses routed inside a fuselage section. Violations indicated by the red circles. Bottom: Refinement result with all violation removed
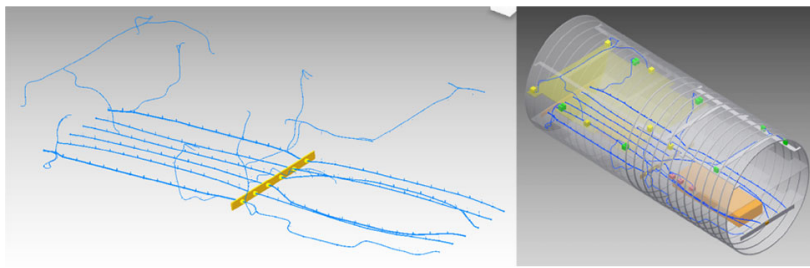

Fig. 19 View of multiple wire harnesses, routed in two contiguous fuselage sections, connecting at the production breaks. View with (right) and without (left) displayed routing environment

are present, but in the second case (right), the objective functions, in both the Initialization and Optimization step, are positively affected when the harnesses are located in the reserved space.

Figure 21 top shows the capability of the tool to handle the minimum bend radius constraint. Figure 21 bottom show the ability of the routing system to deal with grey areas, in this case a hot zone: note how the cost of the harness routed through the hot area (end), even with the required extra protection, results lower than the harness (start) routed outside the hot zone. Changing the heat intensity of the heat source or the cost of the heat protection covering, might lead to different results.
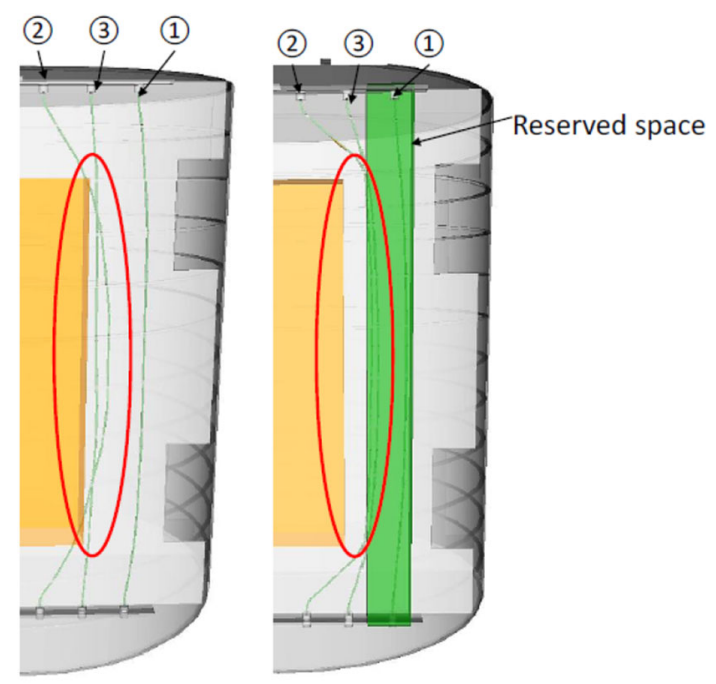

Fig. 20 Harness routing results with (right) and without (left) consideration of the reserved space

Figure 22 demonstrates the capability of the tool to update the routing result when the routing environment is changed. In this case an obstacle has been added. Since this change did not require any road map regeneration, the optimization process could simply be re-executed without significant DMU pre-processing work. In case of changes affecting surfaces where the harness can attach, at least a partial regeneration of the road map can be necessary, before running the optimization. This would increase the computation time, but considering the achieved level of automation, even such changes can be easily accommodated.

\section{Conclusions and recommendations}

In this paper, a method is proposed to handle the $3 \mathrm{~d}$ routing process of wiring harnesses as an optimization problem. This allows the full automation of one of the most time consuming processes in the physical design phase of the EWIS. Due to the multi-origin/multi-destination feature, current pathfinding processes used, for example, in car navigation, are not applicable here. In this case, a two-step, hybrid optimization strategy has been developed, where the harness breakouts and clamping points are used as waypoints to route the harnesses in any given geometrical environment. In the first step, (Initialization) the number of clamping points and their initial position is determined using a multilevel optimization; in the second step (Refinement), the second optimization process moves the clamping points to find minimum cost harnesses, without any constraint violation. 


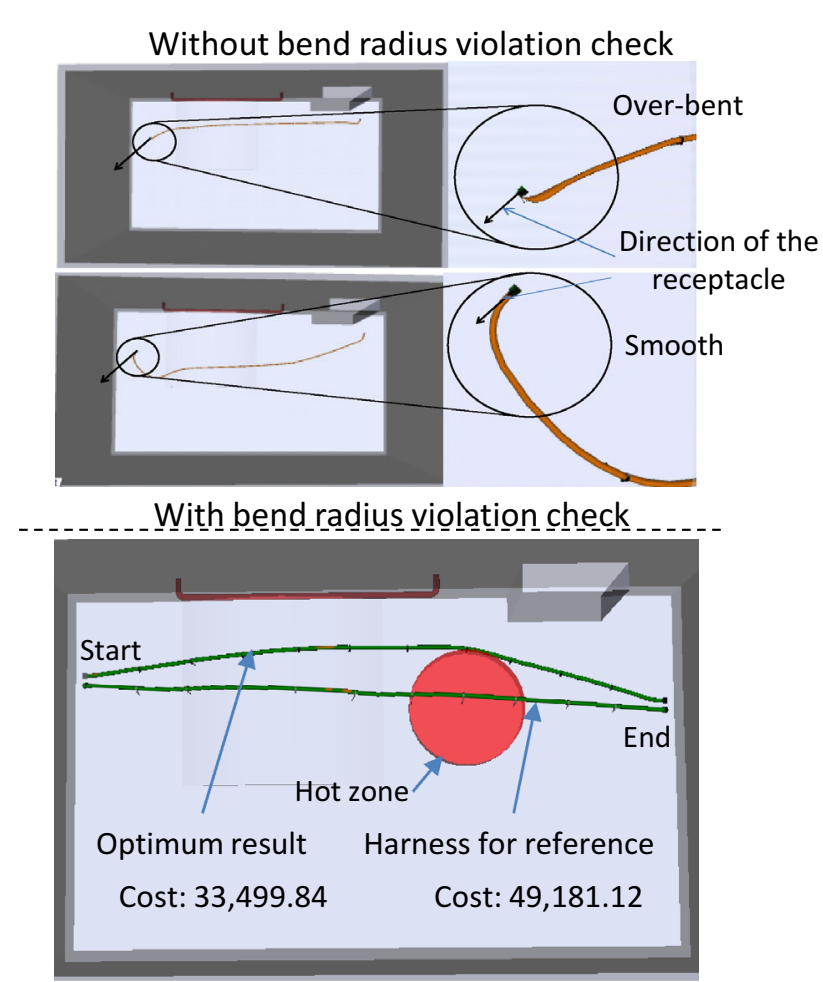

A detour is taken for the minimum cost

Fig. 21 Capability to handle the bend radius violation (top) and hot zone (bottom)

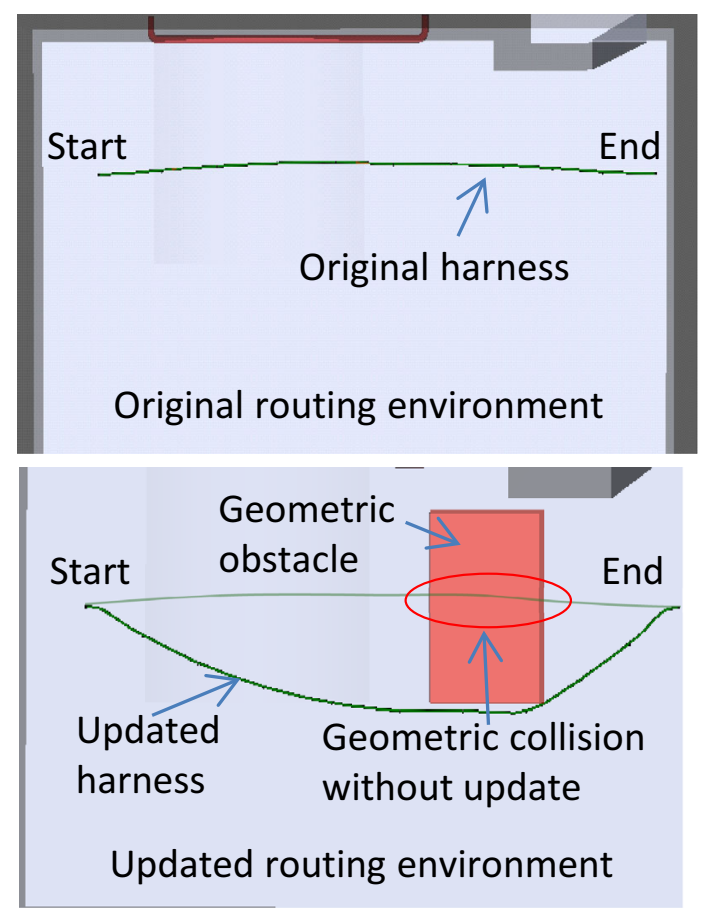

Fig. 22 Update the routing result when the routing environment is updated (i.e., adding a geometric obstacle)
The optimization constraints are used to capture the various design rules implemented by EWIS manufacturers in their current practice and those prescribed by certification authorities to guarantee safe installations. A limited but representative set of these rules/constraints has been selected for the tool demonstrator discussed in this paper. These include design rules such as no geometric collisions, no bend-radius violations, minimum and maximum clamping and fixing distances. They also include special rules to deal with EWIS routing in critical environmental areas, such as hot and flammable areas. The KBE system used to develop the proposed routing system provided all the required rule-based geometry generation and manipulation methods necessary for the implementation of the mentioned rules.

The selected design rules are all modelled and handled as independent modules. This makes the proposed approach fully scalable. In a future development of the HDEE, other rules related to, for example, manufacturing, installation, maintenance, inspection, and so forth could be included in the same way as the ones currently implemented, without significant changes on the main system architecture and overall process workflow.

The HDEE has been tested on a number of relevant study cases and has demonstrated the ability to achieve fully automatic 3D routing and mitigate the risk of lastminute changes in the routing environment. In view of deploying the proposed system into an industrial setting, the following extensions and further investigation are recommended.

In the current implementation, the produced harness routings are only optimized for cost. It would be useful to extend the HDEE capability such to allow multi-objective optimization and produce, for example, Pareto fronts of cost/weight solutions. By simply modifying the cost coefficients (all exposed in the HDEE setting files) of the current objective function, it is already possible to optimize the routing for weight. By introducing some coefficient, such as the classical "cost of a pound" and allowing some longer computational time, multi-objective optimization studies could be performed.

The studies performed in this work have been all performed using a conventional desktop computer, without exploiting any parallel computing opportunity yet. The multilevel optimization approach used in the Initialization phase, however, could be easily accelerated using parallel computing. Also, the possibility to route more harnesses in parallel, rather than sequentially as now, would enable not only gains in terms of computation time, but also to achieve better global results.

Although the HDEE provides already a basic user interface to check and control the automation process, for a 
future industrial application, it is recommended to add new methods and strategies to give designers more interaction and control on the routing process performed by the optimizer. For example, it would be convenient to allow the designer interrupting the automatic routing process to interactively move or fix given clamping points, or anyhow to steer the design as convenient.

Acknowledgements The authors express their gratitude to John van Lugtenburg, Simon Taylor, and Tobie van den Berg from Fokker Elmo B.V. for their precious advices and continuous support.

Open Access This article is distributed under the terms of the Creative Commons Attribution 4.0 International License (http://crea tivecommons.org/licenses/by/4.0/), which permits unrestricted use, distribution, and reproduction in any medium, provided you give appropriate credit to the original author(s) and the source, provide a link to the Creative Commons license, and indicate if changes were made.

\section{References}

1. Airliners.: A380 Electrical Harnesses. http://www.airliners.net/ aviation-forums/tech_ops/read.main/157173/\#menu77 (2012). Accessed 06 March 2012

2. Wong K.: What Grounded the Airbus A380? http://www.cada lyst.com/management/what-grounded-airbus-a380-5955 (2006). Accessed 06 March 2012

3. Safran Group.: More Electric Aircraft. http://www.safran-group. com/site-safran-en/innovation-429/areas-of-expertise/more-elec tric-aircraft/ (2014). Accessed 25 Sep 2014

4. Federal Aviation Administration, 43.13-1B-Acceptable Methods, Techniques, and Practices-Aircraft Inspection and Repair (1998)

5. Conru, A.B.: A genetic approach to the cable harness routing problem, in Evolutionary Computation, 1994. IEEE World Congress on Computational Intelligence., Proceedings of the First IEEE Conference on 1994. pp 200-205

6. Ong, N.S.: Activity-based cost tables to support wire harness design. Int. J. Prod. Econ. 29(3), 271-289 (1993)
7. Billsdon, R., Wallington, K.: Wiring harness design: can a computer help. Comput. Control Eng. J. 9(4), 163-168 (1998)

8. Ritchie, J.M., et al.: Cable harness design, assembly and installation planning using immersive virtual reality. Virtual Real. 11(4), 261-273 (2007)

9. van der Velden, C., et al.: An intelligent system for automatic layout routing in aerospace design. Innovations Syst. Softw. Eng. 3(2), 117-128 (2007)

10. La Rocca, G.: Knowledge based engineering: between AI and CAD. Review of a language based technology to support engineering design. Adv. Eng. Inform. 6(2), 159-179 (2012)

11. Martins, J.R.R.A., Lambe, A.B.: Multidisciplinary design optimization: a survey of architectures. AIAA J. 51(9), 2049-2075 (2013)

12. U.S. Department of Defense.: MIL-W-5088L. Wiring, Aerospace Vehicle (1991)

13. Federal Aviation Administration.: Aircraft Electrical Wiring Interconnect System (EWIS) Best Practices, Aircraft EWIS Practices Job Aid 2.0

14. La Rocca, G., van Tooren, M.J.L.: Knowledge-based engineering approach to support aircraft multidisciplinary design and optimization. J. Aircr. 46(6), 1875-1885 (2009)

15. La Rocca, G., van Tooren, M.J.L.: Development of Design and Engineering Engines to Support Multidisciplinary Design and Analysis of Aircraft, in Delft Science in Design-A Congress on Interdisciplinary Design, pp 107-124 (2005)

16. La Rocca, G., van Tooren, M.J.L.: Enabling distributed multidisciplinary design of complex products: a knowledge based engineering approach. J. Des. Res. 5(3), 333-352 (2007)

17. Yang, T.A.: Path planning and evaluation in IVHS databases, in Vehicle Navigation and Information Systems Conference (1991)

18. Hartley, T.P., Mehdi, Q.H.. In-Game Adaptation of a Navigation Mesh Cell Path. In Computer Games (CGAMES), 2012 17th International Conference (2012)

19. Padhy, N.P.: Artificial Intelligence and Intelligent Systems. Oxford University Press, Oxford (2005)

20. Fattah, M. et al.: Smart hill climbing for agile dynamic mapping in many-core systems. In: Design Automation Conference (DAC), 2013 50th ACM/EDAC/IEEE (2013)

21. Michalewicz, Z., Fogel, D.B.: How to Solve It: Modern Heuristics. Springer, Berlin (2013)

22. Audet, C., Dennis, J.E. Jr.: Analysis of generalized pattern searches. SIAM J. Optim. 13(3), 889-903 (2002) 